S. Neser

T. Palberg

C. Bechinger

P. Leiderer

\section{Direct observation of a buckling transition during the formation of thin colloidal crystals}

S. Neser $(\bowtie) \cdot C$. Bechinger $\cdot$ P. Leiderer Fakultät für Physik

Universität Konstanz

78434 Konstanz, Germany

\section{T. Palberg}

Institut fǜ Physik

Universität Mainz

55099 Mainz, Germany

\begin{abstract}
We have investigated a colloidal suspension in a thin wedge formed by two glass plates in the presence of a lateral pressure. Starting with a single hexagonal layer, with increasing separation between the glass plates additional layers are added. This process is accompanied by a number of structural transitions necessary to maintain a high packing fraction under the given boundary
\end{abstract}

conditions. Besides the well-known sequence of hexagonal and quadratic phases, we observe two new phases which are identified with the buckling and the rhombic phase recently predicted by other authors.

Key words Colloidal crystals - finite size effects - buckling - layering phase transitions
Suspensions of small colloidal spheres are widely used model systems for crystallization phenomena in two and three dimensions $[1,2]$. There are several methods to prepare two-dimensional (2D) colloidal systems, for example, by confinement of the suspension in a narrow gap between two glass plates [2] or by trapping the particles at the water-air interface [3]. In contrast to ideal 2D systems, for example, electrons in surface states above the liquid helium surface [4], in colloidal systems the dimensionality has to be reduced artificially. It is clear that such a system can only be an approximation of the ideal 2D case. On the other hand, in a colloidal system confined between two glass plates the restriction of motion perpendicular to the $2 \mathrm{D}$ plane can be controlled via particle-wallinteractions and the separation of the walls [5]. Therefore, such a system is ideally suited for investigations of the transition regime between 2D and 3D. Pieranski et al. [6] and van Winkle and Murray [7] investigated colloidal particles in a wedge geometry. Starting with one hexagonal monolayer at small plate separations, with increasing cell height they found a sequence of morphological transitions of the form

$$
n \triangle \rightarrow(n+1) \square \rightarrow(n+1) \triangle,
$$

where $n$ denotes the number of layers, with $\square$ and $\Delta$ corresponding to the quadratic and hexagonal phase, respectively. Koshikiya and Hachisu $[8,9]$ have reported some peculiar maze like patterns in the region between one hexagonal and two quadratic layers. Pansu et al. [10] pointed out that this sequence optimizes the packing fraction of the spheres in the slab and suggested a continuous transition between $n \square$ and $n \triangle$ via an intermediate phase possessing rhombic $(r)$ symmetry. A buckling instability of the 2D hexagonal lattice has been found by Chou and Nelson [11], associated with a second-order phase transition to a buckling phase $(b)$ as a function of increasing cell height. As a result of their Monte Carlo simulations Schmidt and Löwen [12] recently published a phase diagram for hard spheres being confined between two plates with distances ranging between one- and two-particle diameters. They obtained a phase diagram which shows a rich variety of phase transitions including the two new phases, the buckling and the rhombic phase. In this paper we present experimental evidence for both buckling and rhombic phases.

To fabricate cells of wedged geometry, first we thoroughly cleaned microscope cover slides with thicknesses of 2 and $0.17 \mathrm{~mm}$, respectively. Then we put a small droplet 
$(10 \mu l)$ of suspension between the two slides, removed any excess liquid and sealed the sample cell along its perimeter with epoxy glue. As particles we used surfactant stabilized polystyrene spheres (Bangs Labs Lot. No. 20-PS196, $840 \mathrm{~nm}$ diameter by DLS) suspended in water with a volume fraction of $10 \%$ and a polydispersity of about $5 \%$. The solidification of the epoxy sealing and capillary forces causes a bending of the thin cover slide due to mechanical stress and lead to the formation of a wedge between the two glass plates. The resulting geometry of the wedges was determined by analyzing the Newton fringe pattern appearing in the slab.

While the epoxy sealing has a small permeability for water which slowly evaporates out of the cell it is a perfect barricr for the particles. The evaporation of the water gencrates an albeit very small - flow due to which most of the particles move towards the edges of the cell where the particle concentration increases and the system starts to crystallize. This corresponds to applying a lateral pressure to the system. During the evaporation process the phase boundary liquid/solid moves through the cell and a colorful striped pattern develops parallel to the interference fringes of the wedged cell. Typically, the evaporation process takes approximately $2-3$ weeks.

In the following, we will show that those patterns can be interpreted in terms of a sequence of regions with hexagonal and quadratic symmetry. Additionally, we have found different intermediate phases in between, two of them being consistent with the buckling and the rhombic phase predicted by other authors [10-12]. In this paper, we will concentrate on systems which do not exceed two layers.

Starting at a cell height supporting only one hexagonal layer and moving in a direction of increasing cell height we always found the same sequence of phases:

$$
1 \triangle \rightarrow \text { buckling }(b) \rightarrow 2 \square \rightarrow \text { rhombic }(r) \rightarrow 2 \triangle,
$$

where the extent of the regions depends on the actual wedge profile. Figure $1 \mathrm{~A}$ shows a scanning electron microscope picture of the buckling phase taken after complete evaporation of the solvent and removal of the top plate,

Fig. 1A Scaning clectron microscope picture of the buckling phase. $\mathrm{B}$ Buckling phase near the phase boundary to the $1 \Delta$ structure. The cell height increases from right to left. The brighter and darker particles belong to the lower and upper sublayer, respectively and the white lines are a guide to the eye to mark the kinks in the particle chains. Near the $1 \triangle$ structure the kink angle is approximately $120^{\circ}$ C Buckling phase near the phase boundary to the $2 \square$ structure. The cell height increases from right to left. Due to the larger separation of the two sublayers compared to Fig. $1 \mathrm{~B}$, only the particles of the lower sublayer are visible. The white lines are a guide to the eye marking the kinks in the particle chains. In the vicinity of the $2 \square$ structure the kink angles approach 90

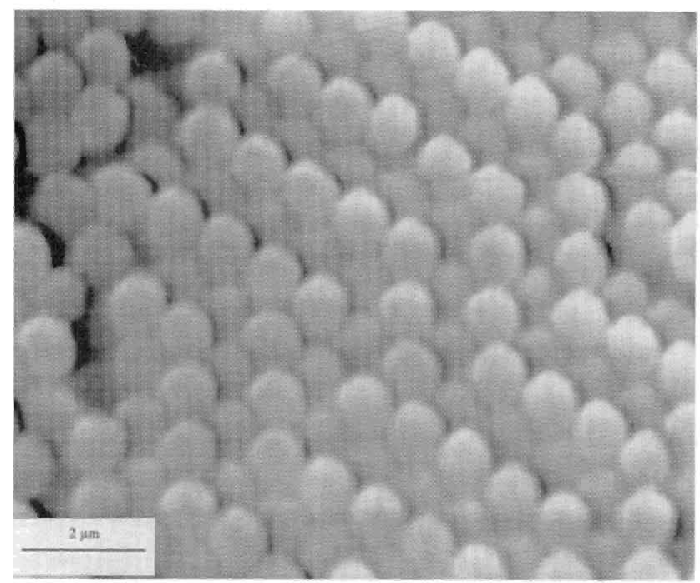

A

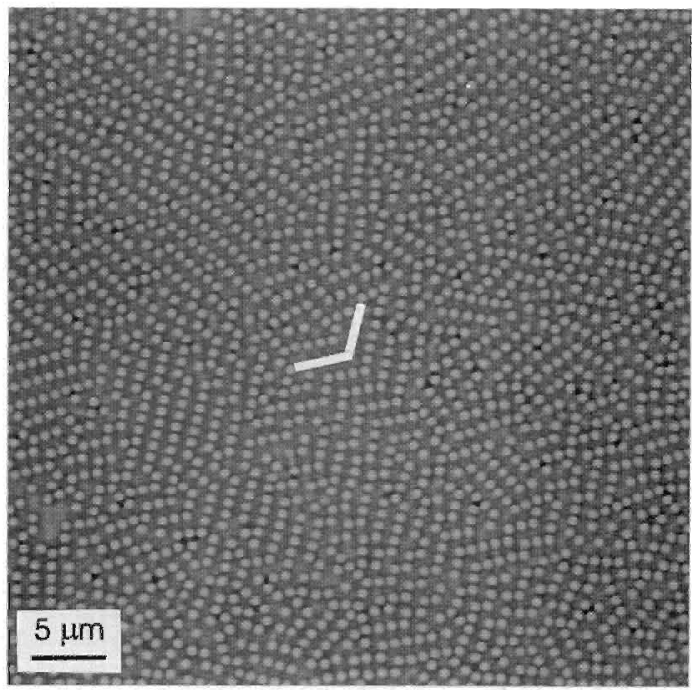

8

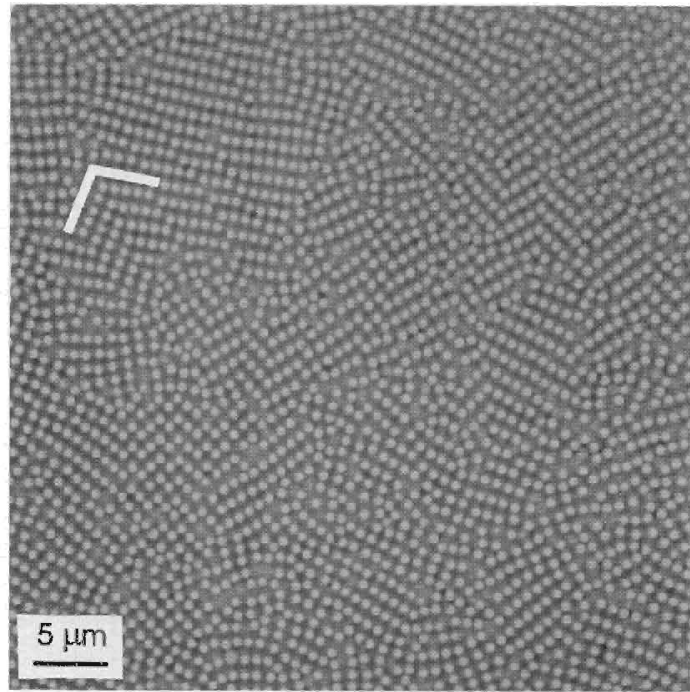

c 
The buckling phase consists of a zig-zag structure of particle chains alternating between an upper and a lower sublayer, vertically separated by a fraction of the particle diameter. Within a chain the particles are closely packed. The separation of neighboring chains belonging to the same sublayer is a function of the cell height. Figure 1B shows a region of the buckling phase near the phase boundary to the $1 \Delta$ structure. The different vertical positions of the upper and the lower sublayer with respect to the focal plane of the microscope gives rise to an optical contrast which allows to distinguish between them. In Fig. 1B the vertical displacement of the sublayers is still small and the distance of two neighboring chains in the same sublayer is close to that of the corresponding distance in the hexagonal monolayer. With increasing cell height the separation of the neighboring chains decreases until it finally approaches the hard sphere radius which is the onset of the quadratic phase. Figure $1 \mathrm{C}$ depicts a region of the buckling phase near the $b \rightarrow 2 \square$ boundary showing the considerably smaller chain separation.

The formation of the buckling phase can be understood in terms of a simple hard sphere packing model. We consider a system with a plate separation of one effective hard sphere diameter subject to lateral pressure. In that case an equilibrium structure of $1 \Delta$ will result, i.e. a densepacked single hexagonal layer. A slight increase in the plate separation will allow the system to release some of the lateral pressure by elevating or lowering particle chains. This corresponds to the buckling instability of the 2D hexagonal lattice shown by Chou and Nelson [12]. A two sublayer system is created in which the lateral chain distance in the upper and lower sublayer, respectively, decreases continuously as the plate separation increases. At the same time the packing fraction is kept at a high level. When $d$ equals the hard sphere diameter the chains in each sublayer touch to form the $2 \square$ structure. We therefore believe to have repeated the experiments of Pieranski et al. [6] and van Winkle and Murray [7] at higher volume fractions where Schmidt and Löwen predict a larger stability region of the buckling phase.

Another dominant feature of the buckling phase are the kinks in the chains. The typical kink angle is $120^{\circ}$ at the onset of buckling decreasing to $90^{\circ}$, when the transition to the two layer quadratic phase is approached. The rather random arrangement of the chains is in good agreement with the miniscule differences in free energy between the possible buckling patterns found by Chou and Nelson [11] and Schmidt and Löwen [12]. As already stated above, with increasing cell height the transition from the buckling to the two layer quadratic phase is reached and a further increase leads to a phase shown in Fig. 2 looking like a distorted hexagonal lattice. These distortions can best be seen following a single lattice line

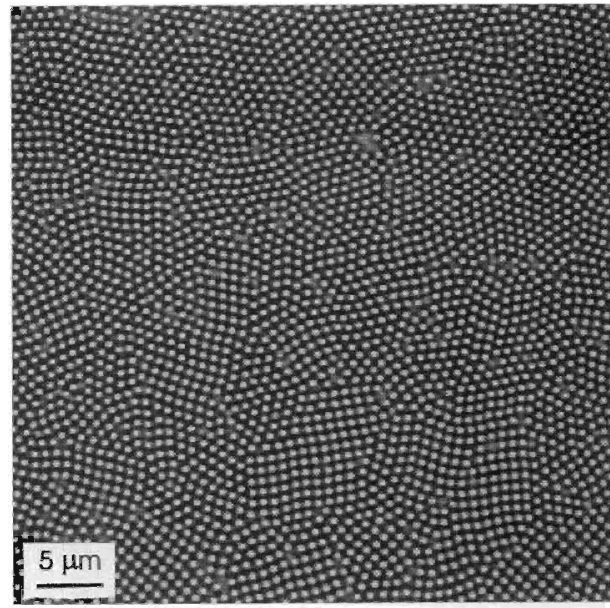

Fig. 2 Distorted hexagonal lattice suggested to be the rhombic phase. The cell height increases from right to left

with the eye. The appearance of that region together with its position between $2 \square$ and $2 \Delta$ structures strongly suggests it to be the rhombic phase. Finally, for sufficient wall separations the two layer hexagonal phase is reached.

It is an interesting question why the buckling and rhombic phases appear so dominantly in our experiment while being only a minor feature in the experiments of other authors $[6,7]$. We suppose that the vertical displacement of chains finally leading to the buckling phase is favored in our geometry, because a lateral flow, i.e. a lateral pressure is imposed during erystallization. This is in good agreement with the results of Schmidt and Löwen, who performed simulation runs with fixed plate separation and packing fraction and observed the presence of a lateral pressure.

In conclusion, we have studied a colloidal system in wedge geometry with a small additional flow. For cell heights between one and two effective hard sphere diameters we find a rather smooth transition from a single hexagonal layer via a buckling phase to two quadratic layers and from those via the rhombic phase to two hexagonal layers. These data are consistent with the recently published theoretical phase diagram of Schmici and Löwen [12]. Both the buckling and the rhombic phase are much more pronounced in our experiments than in those of Pieranski et al. [6] and van Winkle and Murray [7]. We attribute this, in particular, to the presence of an additional flow which forces the particles close to the hard sphere packing limit.

Acknowledgments We gratefully acknowledge financial support of the Deutsche Forschungsgemeinschaft (SFB 306 and SFB 513) and helpful discussions with Hartmut Löwen and Matthias Schmide. 


\section{References}

1. Pusey PN (1991) In: Hansen JP, Levesque D, Zinn-Justin J, Liquids, Freezing and Glass Transition: II, North-Holland, Amsterdam

2. Murray CA, van Winkle DH (eds) (1987) Phys Rev Lett 58:1200-1203

3. Pieranski P (1980) Phys Rev Lett 45: $569-572$

4. Peeters FM (1987) Electrons on a liquid helium film. In: Devreese JT, Peeters FM (eds) Summerschool Proc Advanced Study Institute on the Physics of the Two Dimensional Electron Gas. Plenum. New York
5. Kepler GM, Fraden S (1994) Langmuir 10:2501-2506

6. Pieranski $P$ et al (1983) Phys Rev Let $50: 900$

7. van Winkle DH, Murray CA (1986) Phys Rev A 34:562-573

8. Ogawa T (1983) Phys Soc Jpn 52: $167-170$

9. Kosbikiya Y, Hachisu S (1982) Lecture in Colloid Symp Japan (Sept. 1982) (in japanese language) cited in [8]
10. Pansu B, Pieranski Pi, Pieranski Pa (1984) J Physique 45:331-339

11. Chou T, Nelson DR (1993) Phys Rev E 48:4611

12. Schmidt M, Löwen H (1996) Phys Rev Lett 76:4552 